\title{
\Genome Biology
}

\section{Correspondence \\ Joining forces in the quest for orthologs}

\author{
Toni Gabaldón*, Christophe Dessimoz ${ }^{\dagger}$, Julie Huxley-Jones $\ddagger$, Albert J Vilella§, \\ Erik LL Sonnhammerা and Suzanna Lewis*
}

\begin{abstract}
Addresses: *Bioinformatics and Genomics Programme, Centre for Genomic Regulacion (CRG), Dr. Aiguader, 88. 0800, Barcelona, Spain. †ETH Zurich and Swiss Institute of Bioinformatics, 8092 Zurich, Switzerland. ‡Computational Biology, GlaxoSmithKline Pharmaceuticals, Gunnels Wood Road, Stevenage, Hertfordshire SG1 2NY, UK. \$European Bioinformatics Institute, Wellcome Trust Genome Campus, Hinxton, Cambridge CB10 1SD, UK. TStockholm Bioinformatics Centre, AlbaNova University Centre, Stockholm University, S-106 91 Stockholm, Sweden. ₹Lawrence Berkeley National Laboratory, 1 Cyclotron Road 64R0121, Berkeley, CA 94618, USA.
\end{abstract}

Correspondence: Toni Gabaldón. Email: tgabaldon@crg.es

\begin{abstract}
Better orthology-prediction resources would be beneficial for the whole biological community. A recent meeting discussed how to coordinate and leverage current efforts.
\end{abstract}

Identifying evolutionarily related genes (that is, homologs) is crucial for understanding the nature of genomic diversity and the routes by which it arises. Further, distinguishing homologs into two types, either 'orthologs', genes derived from a common ancestor through speciation, or 'paralogs', those derived through a duplication event, has important implications in studying the evolutionary processes that have shaped a given biological system and, since gene duplication is often associated with processes of functional divergence, for inferring the function of related genes. Indeed, many common research processes depend on accurate orthology predictions, such as finding the gene in a model organism corresponding to a human disease gene, inferring the function of a newly sequenced gene using available experimental assays from its orthologs, inferring species phylogenies by tracing the evolution of orthologous groups, or the characterization of newly sequenced genomes in terms of their encoded genes.

The challenge today, however, is not a lack of orthology predictions, but the plethora of methods and databases that have emerged in recent years (reviewed in [1-3]; additional methods include [4-7]). These methods were developed to meet individual needs - they analyze different datasets, optimize different criteria, and employ different strategies for orthology determination (for example, pairwise comparisons or phylogenetic approaches). Such heterogeneity presents a major obstacle to researchers who simply need to know the current 'best' set of orthologs that can be identified for their gene of interest. Furthermore, the absence of standardized benchmarks and formats makes the integration or comparison of these different orthology datasets extremely challenging and time-consuming.
The field of orthology prediction clearly requires a new momentum that will help resolve these issues and make better use of available resources. Furthermore, this need becomes more urgent when considering the advent of thousands of new genome sequences, facilitated by nextgeneration sequencing technologies. Motivated by this prospect, Erik Sonnhammer and Albert Vilella organized the 'Quest for Orthologs' meeting at the Wellcome Trust Conference Centre in Hinxton, UK in July 2009, to jointly address these issues by bringing together for the first time key representatives of the major methods and databases in the field of orthology prediction.

The participants gathered for this meeting included experts in gene and genome evolution, developers of orthologyprediction algorithms and databases, and curators of model organism databases. The intimate size of the meeting (approximately 30), the varied perspectives, and the sequestered venue created an ideal environment for intensive and fruitful discussions. All participants were given an opportunity to present their work, while still allowing ample time for informal discussion afterwards. In a thought-provoking talk, Bill Pearson (University of Virginia, Charlottesville, USA) confronted the audience head on by questioning the usefulness of orthology. In his view, homology inference is a more reliable indicator of function conservation, as far as purely sequence-based methods are concerned (though he also stressed the inherent limitations of such methods over long evolutionary distances). He noted that function is often conserved between paralogs; and even if not, the potential benefits of distinguishing orthologs from paralogs are outweighed by the risks of inference errors. Teresa Przytycka (National Center for Biotechnology Information, Bethesda, USA) and Ken Wolfe (Smurfit Institute of Genetics, Dublin, Ireland) gave two examples of how augmenting current methods with additional information (protein-protein interaction and synteny data, respectively) might improve predictions. In contrast, most algorithmic 
developments presented at the meeting suggest a general trend toward phylogeny-based orthology inference, a strategy that resembles more closely the original definition of orthology [8]. From all the discussions it was clear that there is a lack of research in the conservation and evolution of protein function. Relevant research will only be possible on the basis of accurately predicted gene histories and functional annotations.

However, the major thrust of the meeting was on identifying points of intersection and the immediate steps that could be jointly undertaken following the workshop to lay the groundwork for the future. As Mike Cherry (Stanford University, Stanford, USA), Pascale Gaudet (Northwestern University, Evanston, USA) and Paul Thomas (SRI International, Menlo Park, USA) explicitly pointed out, and we all clearly recognized, orthologs are essential for more accurately assigning function and the lack of coordination in the field is a major roadblock to progress in this area. Two primary areas for cooperative work were quickly identified: common approaches to benchmark orthology predictions and the formation of standardized protein sets to use as inputs.

\section{Benchmarking orthology-prediction methods coming from alternative methods}

The assessment of orthology-prediction methods is difficult for at least three reasons: first, orthology is defined from the largely unknown evolutionary history of genes, and thus can only be tested indirectly; second, the optimal trade-off between precision and recall strongly depends on the context; and third, the lack of standardized input datasets and data formats are significant practical hurdles to comparing methods. The meeting saw contributions addressing all three issues. David Roos (University of Pennsylvania, Philadelphia, USA) and Adrian Altenhoff (ETH Zurich, Switzerland) introduced novel comparison strategies - based on latent class analysis and species-tree concordance, respectively $[9,10]$ - that complement existing approaches based on gene order and functional conservation [11]. Yet all of these benchmarks rely on assumptions that do not always hold. For instance, genomic rearrangements, recombination, alternative evolutionary histories, or functional divergence among orthologs all disturb these indicators. More importantly, their relative importance strongly depends on the aim of the user. Thus, we not only recognized the usefulness of multiple and at times contradictory criteria, but also the need for a common understanding on their usage and interpretation. Finally, we agreed that adopting a common dataset would eliminate inconsistent use of splicing variants, IDs or data sources and, therefore, greatly facilitate benchmarking.

\section{Standardized protein datasets and file formats}

We identified the ideal common dataset as one that covers a wide spectrum of evolutionary ranges and rates, and that reflects the various common applications of orthology (for example, phylogenetic reconstruction, function prediction, synteny and so on). A working group was established, an initial set of species was proposed (based on earlier work of Paul Thomas, Brigitte Boeckmann and Suzanna Lewis), and it is anticipated that this dataset will be available very soon. Rolf Apweiler (European Bioinformatics Institute, Hinxton, USA) offered that UniProt maintain the set. Regarding the need for standardized input and output data formats, Erik Sonnhammer introduced early specifications of an eXtensible Markup Language (XML) format for orthology, OrthoXML [12], which ignited discussions on potential improvements and compatibility issues with existing methods. Such a format would not only facilitate the comparison of methods, but also their combination. For instance, Roos among others suggested a common web interface to the different predictions methods - similar to HPOC [13] and ProGMap [14], the orthology aggregators presented by Michael Lush (European Bioinformatics Institute, Hinxton, UK) and Jack Leunissen (Wageningen University, the Netherlands), respectively. Several working groups have been set up to further develop these ideas and help their realization. Readers interested in participating should contact us.

In retrospect, a remarkable aspect of this meeting is how few of us, despite our strong common interests and goals, had previously met in person. Yet this is an essential first step for building and coordinating collaborative efforts. Given the positive outcomes of this workshop, we are planning to gather again next year to follow up this work and build on the momentum this meeting generated.

\section{Acknowledgements}

We are grateful to the European Science Foundation and the Wellcome Trust for their generous support, which made this meeting possible, to Pam Garland for coordinating our many requests and making sure we ran on time, to all participants in the meeting for their valuable ideas, and to Michael Ashburner from whom the idea for this valuable workshop also originated.

\section{References}

1. Gabaldón T: Large-scale assignment of orthology: back to phylogenetics? Genome Biol 2008, 9:235.

2. Kuzniar A, van Ham RC, Pongor S, Leunissen JA: The quest for orthologs: finding the corresponding gene across genomes. Trends Genet 2008, 24:539-551.

3. Alexeyenko A, Lindberg J, Perez-Bercoff A, Sonnhammer EL: Overview and comparison of ortholog databases. Drug Disc Today: Technologies 2006, 3:137-143.

4. Datta RS, Meacham C, Samad B, Neyer C, Sjolander K: Berkeley PHOG: PhyloFacts orthology group prediction web server. Nucleic Acids Res 2009, 37(Web Server issue):W84-W89.

5. Jensen LJ, Julien P, Kuhn M, von Mering C, Muller J, Doerks T, Bork P: eggNOG: automated construction and annotation of orthologous groups of genes. Nucleic Acids Res 2008, 36(Database issue):D250-D254.

6. Uchiyama I: MBGD: a platform for microbial comparative genomics based on the automated construction of orthologous groups. Nucleic Acids Res 2007, 35(Database issue): D343-D346. 
7. Roth AC, Gonnet GH, Dessimoz C: Algorithm of OMA for large-scale orthology inference. BMC Bioinformatics 2008, 9:518.

8. Fitch WM: Distinguishing homologous from analogous proteins. Syst Zool 1970, 19:99-113.

9. Altenhoff AM, Dessimoz C: Phylogenetic and functional assessment of orthologs inference projects and methods. PLoS Comput Biol 2009, 5:e1000262.

10. Chen F, Mackey AJ, Vermunt JK, Roos DS: Assessing performance of orthology detection strategies applied to eukaryotic genomes. PLoS One 2007, 2:e383.

11. Hulsen T, Huynen MA, de Vlieg J, Groenen PM: Benchmarking ortholog identification methods using functional genomics data. Genome Biol 2006, 7:R31.
12. OrthoXML [http://www.orthoxml.org]

13. Bruford EA, Lush MJ, Wright MW, Sneddon TP, Povey S, Birney E: The HGNC Database in 2008: a resource for the human genome. Nucleic Acids Res 2008, 36(Database issue):D445-D448.

14. Kuzniar A, Lin $\mathrm{K}, \mathrm{He} \mathrm{Y}$, Nijveen $\mathrm{H}$, Pongor $\mathrm{S}$, Leunissen JA: ProGMap: an integrated annotation resource for protein orthology. Nucleic Acids Res 2009, 37(Web Server issue): W428-W434.

Published: 29 September 2009

doi:10.1186/gb-2009-10-9-403

(c) 2009 BioMed Central Ltd 